\title{
A Tale of Two Biomarkers: The Use of Troponin and CK-MB in Contemporary Practice
}

\begin{abstract}
In its Fall, 2009 issue, Clinical Laboratory Science featured a Focus Series on Cardiovascular Risk Assessment. This series contained articles on B-type natriuretic peptide and genetic markers of cardiovascular disease and explored the links between decreased serum vitamin-D levels and cardiovascular disease. Traditionally, cardiovascular risk assessment has referred to markers that help predict long-term mortality and morbidity for heart disease. Markers like LDL, HDL, triglycerides, total cholesterol, hsCRP, BNP and many others make up this group. Acutely however, in cases of suspected myocardial infarction, cardiovascular assessment relies on a very different class of serum markers. Measurement of CK-MB and troponin proteins allows us to confirm or rule out damage to the myocardium. These markers are in obvious contrast to risk markers that are used as tools to assess a person's future risk for cardiovascular events. When considering markers for myocardial infarct, laboratorians know that $\mathrm{CK}-\mathrm{MB}$ and troponin are both used to give diagnostic and sometimes even prognostic information. In this article, which is an extension of the Fall 2009 Focus series, we review the progress of these two myocardial markers while contemplating the question, is it time to phase-out CK-MB testing given the advances and advantages of troponin testing?'
\end{abstract}

AMY K SAENGER

Clin Lab Sci 2010;23(3):134

Amy K. Saenger, PhD, DABCC, Laboratory Medicine and Pathology Mayo Clinic 200 First Street SW Rochester, MN 55905

Address for Correspondence: Amy K. Saenger, PhD, $D A B C C$, Director, Central Clinical Laboratory/Central Processing, Assistant Professor, Laboratory Medicine and
Pathology Mayo Clinic 200 First Street SW Rochester, MN55905 507-266-6494 saenger.amy@mayo.edu

Modern diagnosis of myocardial infarction includes the use of blood biomarkers, which can also be used for risk assessment and for guiding interventional and noninterventional therapies. An ideal marker of cardiac necrosis should exhibit the following characteristics: cardiac specificity, tissue sensitivity, early and stable release after necrosis, predictable clearance, and the ability to be quantitatively and rapidly measured by cost effective methodologies available in the majority of clinical laboratories. In addition, the biomarkers should provide additional novel information pertinent to clinical care of the patient. This review provides an overview of the use of creatine kinase MB (CK-MB) and cardiac troponin ( $\mathrm{cTn}$ ) in contemporary clinical practice.

\section{Clinical Efficacy of Creatine Kinase MB}

The first cardiac biomarker to fit many of the above criteria was CK-MB. CK-MB is one of three isoenzymes arising from total creatine kinase (CK). Total $\mathrm{CK}$ is a dimeric enzyme composed of two subunits, termed " $M$ " to denote muscle origin or " $\mathrm{B}$ " to designate brain origin. There are three major $\mathrm{CK}$ isoenzymes; the two homodimers CK-MM and CK-BB and the heterodimer CK-MB. ${ }^{1}$ Myocardium is the only tissue that has a high proportion and concentration of CK-MB (Table 1). Release of CK-MB only occurs upon death of myocardial cells and it is not released in the setting of ischemia. ${ }^{2}$ However, CK-MB is also present in skeletal muscle and interpretation of the CK-MB activity assay may therefore be confounded with false positives in the setting of suspected cardiac disease with concomitant skeletal muscle disease. ${ }^{3-6}$ 


\section{CLINICAL PRACTICE}

Table 1: Tissue Distribution of CK Isoenzymes

\begin{tabular}{lccc}
\hline & CK-1 & CK-2 & CK-3 \\
& $(\mathrm{BB})$ & $(\mathrm{MB})$ & $(\mathrm{MM})$ \\
Skeletal & 0 & 1 & 99 \\
Cardiac & 1 & 20 & 79 \\
Brain & 97 & 3 & 0 \\
\hline
\end{tabular}

In the 1970s and 1980s, CK-MB transformed the diagnosis and treatment of patients with acute cardiac events. ${ }^{7} \mathrm{CK}-\mathrm{MB}$ proved more specific than an accurate clinical history, which is often unattainable in the critically ill or is atypical in the elderly and diabetics. CK-MB was more reliable than ECG pattern recognition which can be blind to disease depending on the location of the ischemia. CK-MB also improved specificity over myoglobin ( $90 \%$ vs $70 \%$ specificity, respectively) and consequently became the gold standard for identification of cardiac injury. Myocardial infarction was rarely diagnosed in the absence of CKMB elevation ${ }^{8,9}$ and utilization allowed earlier diagnoses, permitted an uncomplicated mechanism for detection of reinfarction, encouraged the concept of serial measurements, and proved to be one of the first accurate ways to measure infarct volume.

The initial CK-MB assays quantitated activity, but were analytically imprecise and required a significant amount of manual technical intervention. Development of CKMB immunoassays led to increased analytical precision, improved clinical sensitivity (approximately 90\%, dependent upon the assay utilized), and allowed for earlier detection of elevated CK-MB concentrations following an acute myocardial infarction (AMI). However, use of immunoassays raised the frequency of CK$\mathrm{MB}$ interference by acute and chronic skeletal muscle injuries because the dilutions required for activity assays were eliminated. ${ }^{10-12}$ Application of the percent relative index (RI), as defined below, aided in the interpretation of CK-MB values when only cardiac injury or skeletal muscle injury occurred but generated inaccurate diagnoses in patients presenting with concurrent cardiac and skeletal muscle injuries.

$$
\text { Relative Index }=\frac{\mathrm{CK}-\mathrm{MB} \text { (mass) } \times 100}{\mathrm{CK} \text { (activity) }}
$$

Macrokinases, broadly defined as either an immuneglobulin complex with CK-BB or mitochondrial CK, were also discovered to interfere with immunoassays and led to further attempts at CK-MB assay standardization. $^{13,14}$ Although partially successful, poor standardization still exists and results cannot be easily compared over time between manufacturers or platforms.

In the absence of myocardial infarction, CK-MB may be elevated due to poor specificity in patients who present with multiple co-morbidities or conditions including renal failure, non-cardiac surgery, chest trauma, asthma, pulmonary embolism, chronic and acute muscle disease, head trauma, hyperventilation, and hypothyroidism. ${ }^{13,15,16}$ Any elevation in CK-MB becomes confounded with questionable analytical and biological interferences and restricts the use of CK-MB as a stand alone diagnostic cardiac marker. In addition, reporting $\mathrm{CK}-\mathrm{MB}$ properly requires the use of genderspecific reference ranges, which many laboratories fail to do. ${ }^{17}$ By mid-1990 it was evident that better biomarkers were needed to improve patient care and quality outcomes.

\section{The Advent of Troponin}

Development of cardiac troponin I (cTnI) and troponin $\mathrm{T}(\mathrm{cTnT})$ assays in the 1990s revolutionized the use of biomarkers for diagnosis and risk stratification of cardiac injury. Troponin consists of three subunits: T, I, and $\mathrm{C}$ (Figure 1). Troponin $\mathrm{T}$ is a protein that binds tropomyosin; troponin I enhances the interaction of actin and myosin in the myocardium, and troponin $\mathrm{C}$ is responsible for calcium binding within the complex and results in muscle contraction. Troponin $\mathrm{C}$ is present in both the myocardium and skeletal muscle whereas troponin $\mathrm{T}$ and I are highly specific for cardiac muscle. There is a cytosolic pool of unbound cardiac troponin which is released acutely after myocardial infarction, and mimics the release of other cytosolic proteins like CK. The smaller cytosolic pool represents about $6 \%$ of cTnT and $3 \%$ of cTnI, and a similar percentage of the cytosolic pool exists for CK-MB. The increased sensitivity of cardiac troponin over CK-MB is primarily due to the fact that the percentage of troponin released into the blood after an acute cardiac event is greater for troponin than CK-MB. ${ }^{4,18}$ There is a $13-15$ fold 


\section{CLINICAL PRACTICE}

increase in troponin per gram of myocardium that is primarily complexed and this complexed form is responsible for the late and not early release of troponin. ${ }^{19-22}$ Troponin elevations persist in the blood due to the slow release and degradation of the structural pool, and the half-life of complexed troponin is approximately 2 hours.

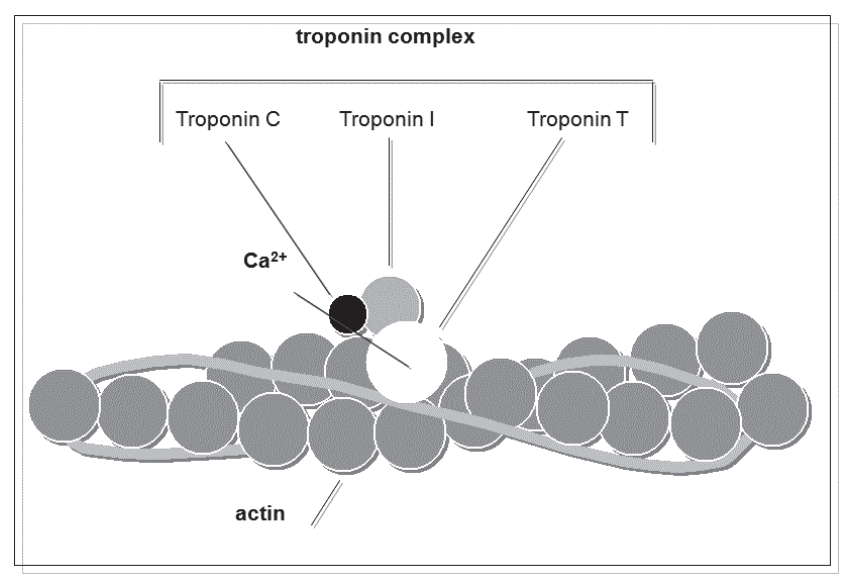

Figure 1: Structure of Troponin

In 2000 the European Society of Cardiology (ESC) and American College of Cardiology (ACC) task force concluded that diagnosis of AMI required biochemical evidence of necrosis and indicated the marker of choice was troponin. ${ }^{23}$ Recommendations were also put forth to utilize troponin for risk stratification and CK-MB was advocated at that time to be measured in conjunction with troponin to distinguish acute MI from an older injury due to troponin persistence. In 2000, there were problems with the analytical aspects of troponin assays which included poor precision, poor standardization, and an overall lack of harmonization, all of which prevented troponin from being recommended as a stand-alone biomarker.

There is only one manufacturer of the troponin $T$ assay (Roche Diagnostics), but there are numerous companies that market troponin I. The antibodies for each troponin I assay are directed towards different epitopes and each manufacturer has a different calibration standard. These dissimilarities result in troponin I assays that have different recommended cutoffs, precision profiles, and positive predictive values. Lack of standardization among troponin I assays remains a source of confusion and potential misclassification of patients by clinicians who utilize troponin I results from different laboratories, a common example being an emergency room point-of-care method versus an automated core laboratory result. Analytical issues remain an unsolved problem for many laboratories, and the majority of assays still suffer from overall imprecision at very low concentrations, including most point-of-care assays.

Troponin guidelines were updated in 2007 by both clinical (ACC/ESC/AHA) and biochemical (NACB) expert groups to recommend use of a troponin concentration greater than the $99^{\text {th }}$ percentile for diagnosis of acute myocardial infarction. ${ }^{24,25}$ The International Federation of Clinical Chemistry and Laboratory Medicine recognized the definition of the $99^{\text {th }}$ percentile as the upper limit of normal for troponin. Accepted by the ESC and ACC, the $99^{\text {th }}$ percentile reference interval for any troponin assay is the $99^{\text {th }}$ percentile generated from a presumably normal reference population (mean \pm 3 standard deviations) and should be used as the cutoff for determining abnormal values. Analytical guidelines recommend an optimal coefficient of variation for troponin of $<10 \%$ at the $99^{\text {th }}$ percentile. In 2009, only three assays meet these precision criteria.

The formal definition from the 2007 guidelines specifies evidence of a changing (delta) pattern of cardiac markers (preferably troponin) with at least one value above the $99^{\text {th }}$ percentile reference range. However there has been substantial confusion about use of the $99^{\text {th }}$ percentile and a lack of understanding that a single elevated troponin above the $99^{\text {th }}$ percentile does not diagnose AMI; instead, a pattern of troponin rise and fall is required. There are trends toward use of ultrasensitive troponin assays, which may be achieved by longer sample incubation times or enhanced signal amplification techniques. Use of ultrasensitive troponin assays may result in a 10 -fold lower reportable concentration and allows for the potential to identify AMI patients at earlier time points. ${ }^{24}$

As assays have progressively increased in sensitivity, it is clear that even the most minor elevations are indicative of cardiac damage (acute or chronic) and thus are 


\section{CLINICAL PRACTICE}

significant and predictive for patients. This can make the differential diagnosis difficult in some patients who may not have a clear etiology for a chronically increased troponin. Nonetheless, it is clear from several studies that any troponin concentration greater than the $99^{\text {th }}$ percentile defines subsequent risk. ${ }^{26-30}$ While an elevation of cTnT or cTnI is specific for cardiac injury, there are rare exceptions of analytical false positives most often due to fibrin interference or cross reacting antibodies. ${ }^{31}$ These interferences are less frequent with current generation assays as technologies have substantially improved over the last decade. The majority of interferences are eliminated by recentrifuging specimens, performing dilution studies, or using heterophile antibody blocking tubes.

Troponin assays have substantially improved analytically in terms of precision, accuracy, and detection limits, which have enhanced the clinical utility for earlier diagnosis of AMI, reinfarction, and risk prediction for future cardiac events. The use of troponin has gradually superseded CK-MB in the majority of indications and patients.

\section{Troponin versus CK-MB}

It has been observed and well-documented that troponin concentrations rise quickly after the onset of chest discomfort. Thus, in upwards of $80 \%$ of patients, a definitive diagnosis can be made in a 2-3 hours using only troponin. ${ }^{32}$ The previously touted "rapidly rising" markers which lack specificity such as myoglobin, CK$\mathrm{MB}$, and fatty acid binding protein are no longer needed. ${ }^{33-35}$ Values of both CK-MB and troponin generally peak within 24 hours or even earlier when reperfusion therapies are effective. For these reasons, CK-MB is not used definitively for diagnosis without troponin. When troponin is below the $99^{\text {th }}$ percentile but the CK-MB is elevated, the patient prognosis is excellent. ${ }^{36}$ However, the opposite statement does not hold any validity. Individuals with an elevated troponin but not CK-MB still have a marked increased risk for development of future coronary events, morbidity, and mortality. 28,32 A rising or falling pattern of cardiac troponin provides all the pertinent information needed to time, diagnose, and treat acute events and CK-MB does not provide any supplemental knowledge to the physician.
Prognosis after acute myocardial infarction is closely related to the extent of myocardial damage. Calculations based on analysis of serial levels of CK or CK-MB have provided estimate of the infarct size for many years since the pioneering work of Sobel and Roberts. ${ }^{37-40}$ For clinicians, peak concentrations provide a reasonable clinical estimate if a sufficient number of data points are collected and if an accurate assessment of whether reperfusion (which changes the kinetics and magnitude of CK-MB release) has occurred. Although peak concentrations may relate to overall subsequent cardiovascular risk, there are major limitations in using these measurements to quantify infarct size.

Recent data suggest that troponin concentrations provide better estimates of infarct size. Ingkanisorn et $a t^{41}$ published the first work investigating the association between early troponin concentrations and infarct size in patients with acute coronary syndromes. They reported that the peak troponin I correlated with acute infarct mass in patients undergoing acute primary percutaneous coronary intervention $(\mathrm{PCI})(\mathrm{r}=0.83, p$ $<0.001, n=23$ ). These data were confirmed in a study by Younger et $a^{42}$ where there was a significant relationship between infarct size and cTnI in both thrombolyzed and non-thrombolyzed patients. Troponin provides better information than CK-MB on infarct size and thus CK-MB should not be ordered for this purpose.

Recent data on utilization of cardiac biomarkers for diagnosis of reinfarction are sparse, largely because modern day contemporary intervention for AMI and suspected AMI is rapid, aggressive, and predicated on the use of troponin. Some argue that CK-MB should still be used to diagnose reinfarction but supportive data for troponin demonstrates equivalency. The 2007 ACC/ESC/AHA guidelines still suggest that serial samples for CK-MB may be helpful, yet advocacy for the use of CK-MB for reinfarction diagnosis likely represents a widespread level of comfort with CK-MB rather than evidence based data. The studies which originally validated CK-MB for diagnosis of reinfarction were conducted at a time prior to the extensive use of interventional strategies for AMI. Patients were often hospitalized for several days and recurrent chest discomfort rarely triggered coronary angiography and/or 


\section{CLINICAL PRACTICE}

intervention. Reinfarction criteria still required a rise from the prior baseline; this information was readily accessible from commonly obtained surveillance CK$\mathrm{MB}$ values. The criteria proposed for use of CK-MB in reinfarction ${ }^{43}$ and modified by many clinical trial groups have never been validated. Literature comparing CK$\mathrm{MB}$ and troponin simultaneously in reinfarction is minimal, with the most comprehensive paper stemming from Apple et al. ${ }^{44}$ Troponin provides equivalent, if not superior, information to CK-MB in patients who delay acute intervention and a $20 \%$ change in troponin is required for diagnosis. Awareness surrounding the occasional patient who will have a secondary rise in troponin (especially cTnT) after an ST-segment elevation myocardial infarction, most often in the absence of symptoms, is essential.

CK-MB has also been promoted as providing crucial information in patients undergoing interventional procedures, such as percutaneous coronary intervention (PCI). While it is true that biomarker monitoring is of importance in interventional patients, cardiac intervenetional experts now advocate changing from $\mathrm{CK}-\mathrm{MB}$ to a troponin standard. ${ }^{45}$ However, elevation in CK-MB or troponin retains no prognostic importance in patients who undergo PCI when the baseline CK-MB/troponin is used for risk stratification, ${ }^{46,47}$ suggesting neither marker is specifically prognostic. An elevated troponin can be used to direct specific anticoagulant, anti-platelet and invasive therapies predicated on results from multiple large randomized clinical trials, an important caveat to its use in interventional procedures. ${ }^{25,48}$

Troponin concentrations can be elevated in many situations but observed commonly in renal failure patients, the critically ill, and with strenuous exercise (Table 2). These chronic elevations are not "false positives" but instead provide prognostic significance. $\mathrm{CK}-\mathrm{MB}$ testing is not useful in these populations, as CK-MB will likely also be elevated and should not be retained for this purpose. ${ }^{16,49}$ In contrast to chronic troponin elevations, chronic CK-MB elevations have not been reported to help distinguish risk for further adverse outcomes. Therefore, although renal patients can have a chronically elevated troponin, measurement of troponin should still supersede CK-MB testing to predict risk of cardiovascular events. Ultimately, it is evident that a rising or falling pattern of troponin can be used to differentiate those with acute disease from those with chronic troponin elevations.

Table 2: Troponin Elevations in Patients Without Overt Ischemic Heart Disease

Acute rheumatic fever
Amyloidosis
Cardiac trauma (including
contusion, surgery, ablation)
Cardiotoxicity from
chemotherapy
Chronic renal failure
Heart failure
Critically illness
Diabetes
Drug toxicity
Hypertension

Hypotension, often with arrhythmias Hypothyroidism Inflammatory disease (including myocarditis, bacterial endocarditis, Kawasaki disease) Acute neurological diseases Post-operative non-cardiac surgery Pulmonary embolism, severe pulmonary hypertension Sepsis

\section{Summary}

With the advent of sensitive cardiac troponin assays, CK-MB no longer provides additional information to the diagnostic algorithm for acute myocardial infarction. The ordering patters of CK-MB often represent a clinical level of comfort which may be difficult to supersede. Contemporary troponin testing essentially renders the use of $\mathrm{CK}-\mathrm{MB}$ as obsolete and frequent ordering of CK-MB yields unnecessary costs for the patient and the laboratory. In situations where little, if any, incremental information is provided with duplicate testing laboratories should focus efforts towards educating clinicians and/or implement strategies to restrict CK-MB orders. Troponin assays in 2009 are continually improving in precision, accuracy, sensitivity, and specificity.

\section{REFERENCES}

1. Christenson RH, Azzazy HM. Biochemical markers of the acute coronary syndrome. Clin Chem 1998;44:1855-64.

2. Ishikawa Y, Saffitz JE, Mealman TL, et al. Reversible myocardial ischemic injury is not associated with increased creatine kinase activity in plasma. Clin Chem 1997;43:467-75.

3. Wu AH, Wang XM, Gornet TG, Ordonez-Llanos J. Creatine kinase $\mathrm{MB}$ isoforms in patients with skeletal muscle injury: ramifications for early detection of acute myocardial infarction. Clin Chem 1992;38:2396-400.

4. Adams 3rd JE, Bodor GS, Davila-Roman VG, et al. Cardiac troponin I. A marker with high specificity for cardiac injury. Circulation 1993;88:101-6. 


\section{CLINICAL PRACTICE}

5. Adams 3rd JE, , Schechtman KB, Landt Y, Ladenson JH, Jaffe AS. Comparable detection of acute myocardial infarction by creatine kinase MB isoenzyme and cardiac troponin I. Clin Chem 1994; 40:1291-5.

6. Tsung JS, Tsung SS. Creatine kinase isoenzymes in extracts of various human skeletal muscles. Clin Chem 1986;32:1568-70.

7. Jaffe AS. Biochemical detection of acute myocardial infarction. In: Gersh B, Rahimtoola S, eds. Acute Myocardial Infarction, Elsevier, 1991:110-27.

8. Roberts R. The two out of the three criteria for the diagnosis of infarction: Is it passe? Chest 1984;86:511-3.

9. Lee TH, Goldman L. Serum enzyme assays in the diagnosis of acute myocardial infarction. Recommendations based on a quantitative analysis. Ann Intern Med 1986;105:221-33.

10. Mair J, Artner-Dworzak E, Dienstl A, et al. Early detection of acute myocardial infarction by measurement of mass concentration of creatine kinase-MB. Am J Cardiol 1991;68:1545-50.

11. Collinson P, Rosalki S, Kuwana T, et al. Early diagnosis of acute myocardial infarction by CK-MB mass measurements. Ann Clin Biochem 1992;29:43-7.

12. Bakker A, Gorgels J, van Vlies B, et al. The mass concentrations of serum troponin $\mathrm{T}$ and creatine kinase-MB are elevated before creatine kinase and creatine kinase-MB activities in acute myocardial infarction. Eur J Clin Chem and Clin Biochem 1993;31:715-24.

13. Jaffe AS. Biochemical detection of acute myocardial infarction. In: Gersh B, Rahimtoola S, eds. Acute Myocardial Infarction, Vol. 2nd ed: Elsevier, 1997:136-62.

14. Christenson RH, Vaidya H, Landt Y, et al. Standardization of creatine kinase-MB (CK-MB) mass assays: the use of recombinant $\mathrm{CK}-\mathrm{MB}$ as a reference material. Clin Chem 1999;45:1414-23.

15. Robbins M, Epstein E, Shah S. Creatine kinase subform analysis in hemodialysis patients without acute coronary syndromes. Nephron 1997;76:296-9.

16. Jaffe AS, Ritter C, Meltzer V, et al. Unmasking artifactual increases in creatine kinase isoenzymes in patients with renal failure. J Clin Lab Med 1984;104:193-202.

17. Apple F, Quist H, Doyle P, et al. Plasma 99th percentile reference limits for cardiac troponin and creatine kinase $\mathrm{MB}$ mass for use with European Society of Cardiology/American College of Cardiology consensus recommendations. Clin Chem 2003;49:1331-6.

18. Katus HA, Remppis A, Neumann FJ, et al. Diagnostic efficiency of troponin $\mathrm{T}$ measurements in acute myocardial infarction. Circulation 1991;83:902-12.

19. Adams 3rd JE, Sicard GA, Allen BT, et al. Diagnosis of perioperative myocardial infarction with measurement of cardiac troponin I. N Engl J Med 1994;330:670-4.

20. Apple FS, Falahati A, Paulsen PR, et al. Improved detection of minor ischemic myocardial injury with measurement of serum cardiac troponin I. Clin Chem 1997;43:2047-51.

21. Katus HA, Remppis A, Scheffold $T$, et al. Intracellular compartmentation of cardiac troponin $\mathrm{T}$ and its release kinetics in patients with reperfused and nonreperfused myocardial infarction. Am J Cardiol 1991;67:1360-7.
22. Tanaka H, Abe S, Yamashita T, et al. Serum levels of cardiac troponin I and troponin $\mathrm{T}$ in estimating myocardial infarct size soon after reperfusion. Coron Artery Dis 1997;8:433-9.

23. Alpert JS, Thygesen K, Antman E, Bassand JP. Myocardial infarction redefined--a consensus document of The Joint European Society of Cardiology/American College of Cardiology Committee for the redefinition of myocardial infarction. J Am Coll Cardiol 2000;36:959-69.

24. Apple F, Jesse R, Newby L, et al. National Academy of Clinical Biochemistry and IFCC Committee for Standardization of Markers of Cardiac Damage Laboratory Medicine Practice Guidelines: analytical issues for biochemical markers of acute coronary syndromes. Clin Chem 2007;53:547-51.

25. Morrow D, Cannon C, Jesse R, et al. National Academy of Clinical Biochemistry Laboratory Medicine Practice Guidelines: clinical characteristics and utilization of biochemical markers in acute coronary syndromes. Clin Chem 2007;53:552-74.

26. Antman E, Tanasijevic M, Thompson B, et al. Cardiac-specific troponin I levels to predict the risk of mortality in patients with acute coronary syndromes. N Engl J Med 1996;335:1342-9.

27. Hamm CW, Ravkilde J, Gerhardt W, et al. The prognostic value of serum troponin $\mathrm{T}$ in unstable angina. $\mathrm{N}$ Engl J Med 1992;327:146-50.

28. Babuin L, Vasile V, Rio Perez J, et al. Elevated cardiac troponin is an independent risk factor for short and long term mortality in medical intensive care unit patients. Crit Care Med 2008;36:759-65.

29. Kavsak P, MacRae A, Palomaki G, et al. Health outcomes categorized by current and previous definitions of acute myocardial infarction in an unselected cohort of troponin-naïve emergency department patients. Clin Chem 2006;52:2028-35.

30. Landesberg G, Shatz V, Akopnik I, et al. Association of cardiac troponin, $\mathrm{CK}-\mathrm{MB}$, and postoperative myocardial ischemia with long-term survival after major vascular surgery. J Am Coll Cardiol 2003;42:1547-54.

31. Jaffe AS. Elevations in cardiac troponin measurements: false false-positives: the real truth. Cardiovasc Toxicol 2001;119: 87-92.

32. MacRae A, Kavsak P, Lustig V, et al. Assessing the requirement for the 6-hour interval between specimens in the American Heart Association Classification of Myocardial Infarction in Epidemiology and Clinical Research Studies. Clin Chem 2006;52:812-8.

33. Eggers K, Oldgren J, Nordenskjold A, Lindahl B. Diagnostic value of serial measurement of cardiac markers in patients with chest pain: limited value of adding myoglobin to troponin I for exclusion of myocardial infarction. Am Heart J 2004;148:57481.

34. Ilva $\mathrm{T}$, Eriksson $\mathrm{S}$, Lund $\mathrm{J}$, et al. Improved early risk stratification and diagnosis of myocardial infarction, using a novel troponin I assay concept. Eur J Clin Invest 2005;35:1126.

35. Kavsak P, MacRae A, Lustig V, et al. Effects of contemporary troponin assay sensitivity on the utility of the early markers myoglobin and CKMB isoforms in evaluating patients with 


\section{CLINICAL PRACTICE}

possible acute myocardial infarction. Clin Chem Acta 2007;380:213-6.

36. Goodman S, Steg P, Eagle K, et al. The diagnostic and prognostic impact of the redefinition of acute myocardial infarction: lessons from the Global Registry of Acute Coronary Events (GRACE). Am Heart J 2006;151:654-60.

37. Geltman E, Ehsani A, Campbell M, et al. The influence of location and extent of myocardial infarction on long-term ventricular dysrhythmia and mortality. Circulation 1979;60:805-14.

38. Roberts R, Sobel B, Parker C. Radioimmunoassay for creatine kinase isoenzymes. Science 1976;194:855-7.

39. Roberts R, Henry PD, Sobel B. An improved basis for enzymatic estimation of infarct size. Circulation 1975;52:74354.

40. Smith J, Ambos H, Gold H, et al. Enzymatic estimation of myocardial infarct size when early creatine kinase values are not available. Am J Cardiol 1983;51:1294-300.

41. Ingkanisorn W, Rhoads $\mathrm{K}$, Aletras $\mathrm{A}$, et al. Gadolinium delayed enhancement cardiovascular magnetic resonance correlates with clinical measures of myocardial infarction. J Am Coll Cardiol 2004;43:2253-9.

42. Younger J, Plein S, Barth J, et al. Troponin-I concentration 72 $\mathrm{h}$ after myocardial infarction correlates with infarct size and presence of microvascular obstruction. Heart 2007;93:154751.

43. Muller J, Rude R, Braunwald E, et al. Myocardial infarct extension: occurrence, outcome, and risk factors in the Multicenter Investigation of Limitation of Infarct Size. Ann Intern Med 1988;108:1-6.

44. Apple FS, Murakami MM. Cardiac troponin and creatine kinase $\mathrm{MB}$ monitoring during in-hospital myocardial reinfarction. Clin Chem 2005;51:460-3.

45. Kleiman NS. Measuring troponin elevation after percutaneous coronary intervention: ready for prime time. J Am Coll Cardiol 2006;48:1771-3.

46. Miller WL, Garratt KN, Burritt M, et al. Baseline troponin level: key to understanding the importance of post-PCI troponin elevations. Eur Heart J 2006;27:1061-9.

47. Prasad A, Rihal C, Lennon R, et al. Significance of periprocedural myonecrosis on outcomes following percutaneous coronary intervention: an analysis of pre and post intervention troponin $\mathrm{T}$ levels in 5487 patients. Circulation: Cardiovascular Intervention 2008: in press.

48. Thygesen $\mathrm{K}$, Alpert J, White $\mathrm{H}$, et al. Universal definition of myocardial infarction. Circulation 2007;116:2634-53.

49. Jaffe AS, Garfinkel B, Ritter C, Sobel B. Plasma MB creatine kinase after vigorous exercise in professional athletes. Am J Cardiol 1984;53:856-8.

The peer-reviewed Clinical Practice Section seeks to publish case studies, reports, and articles that are immediately useful, are of a practical nature, or contain information that could lead to improvement in the quality of the clinical laboratory's contribution to patient care, including brief reviews of books, computer programs, audiovisual materials, or other materials of interest to readers. Direct all inquiries to Libby Spence, PhD, CLS(NCA), Clin Lab Sci Clinical Practice Editor, University of Mississippi Medical Center, 2500 North State Street, Jackson, MS 39216, lspence@umc.edu.

Clinical Laboratory Science encourages readers to respond with thoughts, questions, or comments regarding these articles. Email responses to westminsterpublishers@comcast.net. In the subject line, please type the journal issue and lead author such as "CLIN LAB SCI 23(3) RE SAENGER". Selected responses may appear in the Dialogue and Discussion section in a future issue. Responses may be edited for length and clarity. We look forward to hearing from you. 